\title{
Treatment Outcomes in Head and Neck Cancer Patients 80 Years Old and over
}

\author{
Tomonori Terada, Nobuhiro Uwa, Kosuke Sagawa, Takeshi Mohri, Nobuo Saeki, Kota Kida, \\ Kenzo Tsuzuki, Masafumi Sakagami \\ Department of Otolaryngology_-Head and Neck Surgery, Hyogo College of Medicine, Nishinomiya City, Japan \\ Email: t-terada@hyo-med.ac.jp
}

Received 7 September 2015; accepted 16 November 2015; published 19 November 2015

Copyright (C) 2015 by authors and Scientific Research Publishing Inc.

This work is licensed under the Creative Commons Attribution International License (CC BY).

http://creativecommons.org/licenses/by/4.0/

(c) (i) Open Access

\begin{abstract}
Background: With the recent aging of society, the need for medical treatment of elderly patients with head and neck cancer seems to have been increasing. Method: The present study analyzed all 103 patients with head and neck cancer $\geq 80$ years, and we compared results with those of the previous generation (Group P; range: 75 - 79 years) comprising 104 patients treated in the same period. Results: We provided treatment just as wanted and could not choose it often. The reasons were oncological factors such as unresectable tumor or distant metastasis, refusal of treatment, and physical factors such as poor PS or number of comorbidities. Conclusion: Treatment choices should be based on the wishes and motivations of the patient and the medical assessment of physical function. When a patient $\mathbf{\geq 8 0}$ years old is treated, the high incidence of complications and severity of the disease should be considered.
\end{abstract}

\section{Keywords}

80 Years Old and over, Elderly Patients, Head and Neck Cancer, Treatment

\section{Introduction}

With the recent aging of society, the need for medical treatment of elderly patients with head and neck cancer seems to have been increasing. According to global health statistics announced on May 15, 2014 by the World Health Organization (WHO), the Japanese average lifespan was first among the participating 194 countries, at 84 years [1]. By sex, women were also first, at 87 years, and men were fifth at 80 years [1]. The Japanese average lifespan is expected to keep increasing, and Japanese society will thus continue to age, bringing increased opportunities to treat elderly patients with head and neck cancer. Because elderly individuals often have comorbidities and problems with social support in comparison with younger individuals, treatment according to policy may not take place [2] [3]. The present study was therefore performed to evaluate the situation for patients $\geq 80$ 
years old with head and neck cancer.

\section{Patients and Methods}

From January 2000 to December 2011, a total of 918 patients with head and neck cancer visited the Department of Otolaryngology - Head and Neck Surgery at Hyogo College of Medicine. Of these, the present study analyzed all 103 patients $\geq 80$ years old (11.2\%). These patients comprised 81 men and 22 women, and mean age was 83.8 years (range, 80 - 93 years). Primary tumor sites were the larynx (40\%), mesopharynx (17\%), hypopharynx (13\%), oral cavity (13\%), nasopharynx (6\%), salivary glands (6\%), nasal cavity/paranasal sinuses (5\%), and auditory organs (2\%). The present study analyzed TNM stage, performance status (PS), multiple primary cancers, comorbidities, treatment, complications after treatment, and prognosis. In addition, we compared results with those of the previous generation (Group P) comprising 104 patients (95 men, 9 women)with a mean age of 76.7 years (range, 75 - 79 years)treated in the same period of January 2000 to December 2011, to investigate the influences of chronological age.

The Kaplan-Meier method was used to determine survival rates. Statistical analyses were performed using the chi-square test or non-parametric Mann-Whitney's U test. In all cases, values of $\mathrm{p}<0.05$ were regarded as statistically significant.

\section{Results}

\subsection{TNM Stage}

Cancer was often advanced, with Stage III in 18 cases (17.5\%) and Stage IV in 40 cases (41.2\%). The rate of advanced cancer for primary tumor sites was $34.1 \%$ for the larynx, $0 \%$ for the nasopharynx, $82.4 \%$ for the mesopharynx, $92.3 \%$ for the hypopharynx, $53.8 \%$ for the oral cavity, $100 \%$ for the salivary glands, $80 \%$ for the nasal cavity/paranasal sinuses, and 50\% for the auditory organs. On the other hand, in Group P, Stage III was seen in 14 cases (13.5\%), and Stage IV in 47 cases (45.2\%). No significant difference was seen between groups (Table 1).

\subsection{Performance Status (PS)}

We assessed the daily living abilities of patients according to Eastern Cooperative Oncology Group (ECOG) PS at pretreatment [4] (Table 2). PS was 0 in 45 patients (43.7\%), 1 in 34 patients (33.0\%), 2 in 16 patients (15.5\%), 3 in 5 patients (4.9\%), and 4 in 3 patients (2.9\%), and mean PS was 0.9 (Table 1). In Group P, mean PS was 0.63 , and no significant differences were seen between groups.

\subsection{Multiple Primary Cancers}

Multiple primary cancers were present in 41 patients (39.8\%) (Table 1), at a total of 55 sites overlap, and the most common sites were the head and neck, esophagus, lungs, and stomach, with 9 cases each. No significant difference in number of patients with multiple primary cancers was seen between groups.

\subsection{Comorbidity}

We applied the Chalson comorbidity index [5] and Cumulative Illness Rating Scale for Geriatrics (CIRS-G) [6] and chose the comorbidity that might have influenced the original selection of treatment from among nine items: 1) hypertension; 2) ischemic heart disease; 3) cerebrovascular disease; 4) diabetes; 5) respiratory disease (chronic obstructive pulmonary disease, asthma attack, interstitial pneumonia); 6) kidney failure requiring dialysis; 7) liver disease (liver cirrhosis); 8) mental disease (dementia, depression); or 9) hematological disease (myelodysplastic syndrome, idiopathic thrombocytopenic pupura).

Pretreatment comorbidity was found in 88 patients (85.4\%). Hypertension was the most common (59.2\%), followed by ischemic heart disease (33.0\%), cerebrovascular disease (23.3\%), diabetes (16.5\%), respiratory disease (13.6\%), and mental disease (11.7\%). Fifteen patients showed no pretreatment comorbidity (14.6\%), while one comorbidity was present in 32 patients (31.1\%), two in 26 patients (25.2\%), and three or more in 30 patients (29.1\%). A significant difference in the number of comorbidities was evident between the subject group and Group P (p < 0.01) (Table 1). 
Table 1. Patient characteristics in different age groups.

\begin{tabular}{|c|c|c|c|}
\hline Characteristic & Subject group & Group P & Statistically significant \\
\hline Patients $(\mathrm{n}=207)$ & 103 & 104 & \\
\hline Period & $2000-2011$ & $2000-2011$ & \\
\hline \multicolumn{4}{|l|}{ Sex } \\
\hline Male & $81(78.6 \%)$ & $95(91.3 \%)$ & \multirow[t]{3}{*}{$\mathrm{p}<0.01$} \\
\hline Female & $22(21.4 \%)$ & $9(8.7 \%)$ & \\
\hline Age range (mean) & 80 - $93(83.8)$ & $75-79(76.7)$ & \\
\hline \multicolumn{4}{|l|}{ Primary tumor sites } \\
\hline Larynx & 41 & 37 & \multirow{8}{*}{ NS } \\
\hline Nasopharynx & 6 & 4 & \\
\hline Mesopharynx & 17 & 17 & \\
\hline Hypopharynx & 13 & 18 & \\
\hline Oral cavity & 13 & 13 & \\
\hline Salivary glands & 6 & 4 & \\
\hline Nasal cavity/paranasal sinus & 5 & 6 & \\
\hline Auditory organ & 2 & 5 & \\
\hline \multicolumn{4}{|l|}{ TNM stage } \\
\hline Stage I & 20 & 24 & \multirow{4}{*}{ NS } \\
\hline Stage II & 25 & 19 & \\
\hline Stage III & 18 & 1 & \\
\hline Stage IV & 40 & 47 & \\
\hline \multicolumn{4}{|l|}{ Performance status (PS) } \\
\hline PS 0 & 45 & 60 & \multirow{5}{*}{ NS } \\
\hline PS 1 & 34 & 30 & \\
\hline PS 2 & 16 & 8 & \\
\hline PS 3 & 5 & 5 & \\
\hline PS 4 & 3 & 1 & \\
\hline Average PS & 0.9 & 0.63 & NS \\
\hline \multicolumn{4}{|l|}{ Multile primary cancer } \\
\hline None & 62 & 61 & \multirow{4}{*}{ NS } \\
\hline One & 29 & 33 & \\
\hline Two & 10 & 5 & \\
\hline Three or more & 2 & 5 & \\
\hline \multicolumn{4}{|l|}{ Comorbidity } \\
\hline None & 15 & 25 & \multirow{5}{*}{$\mathrm{p}<0.01$} \\
\hline One & 32 & 37 & \\
\hline Two & 26 & 27 & \\
\hline Three or more & 30 & 15 & \\
\hline Average & 1.85 & 1.38 & \\
\hline
\end{tabular}

NS: not significance.

Table 2. Eastern cooperative oncology group (ECOG) performance status.

\begin{tabular}{cl}
\hline Grade & \\
\hline 0 & Fully active, able to carry on all pre-disease performance without restriction \\
1 & $\begin{array}{l}\text { Restricted in physically strenuous activity but ambulatory and able to carry out work of a light or sedentary nature, e.g., light } \\
\text { house work, office work }\end{array}$ \\
2 & Ambulatory and capable of all selfcare but unable to carry out any work activities. Up and about more than $50 \%$ of waking hours \\
3 & Capable of only limited selfcare, confined to bed or chair more than $50 \%$ of waking hours \\
4 & Completely disabled. Cannot carry on any selfcare. Totally confined to bed or chair \\
5 & Dead
\end{tabular}




\subsection{Initial Treatment}

We performed radical standard treatment for 83 patients, palliative treatment for 8 patients, and no treatment at the request of the patient and their family for 12 patients. In Group P, we performed radical standard treatment for 93 patients, palliative treatment for 5 patients, and no treatment for 6 patients. No significant difference was evident between groups (Table 3 ).

Radical standard treatment was not able to be chosen due to oncological factors such as unresectable tumor or distant metastasis in 9 cases, declining treatment (opting to receive no treatment) in 8 cases, and physical factors such as poor PS or numerous comorbidities in 3 cases.

In the 83 cases for which radical treatment was performed, treatment could not be performed according to the policies of our institution in 19 cases (22.9\%), including abbreviation of prophylactic neck dissection, abbreviation of postoperative radiation therapy and abbreviation of combination chemotherapy in radiation therapy. On the other hand, in Group P, patients unable to receive treatment according to our policy comprised only eight of the 93 cases $(8.6 \%)$, showing a significant difference between groups $(\mathrm{p}<0.01)$.

\subsection{Achievement of Planned Initial Treatment}

In the 91 cases in which radical standard or palliative treatment was performed, the planned initial treatment was unable to be achieved in 14 cases (15.4\%), while 77 cases (84.6\%) were able to complete the planned treatment (Table 3). Treatment difficulty was attributed to complications of treatment in eight cases, requests from patients for treatment cancellation in four cases, and physical reasons other than treatment complications in two cases. In Group P, among the 98 cases that received radical standard or palliative treatment, the planned initial treatment was not able to be completed in only 5 cases $(5.1 \%)$, while 93 cases $(94.9 \%)$ were able to complete the planned treatment, representing a significant difference between groups $(\mathrm{p}<0.05)$.

Table 3. Treatment and prognosis in different age groups.

\begin{tabular}{|c|c|c|c|}
\hline Characteristic & Subject group & Group P & Statistically significant \\
\hline Patients $(n=207)$ & 103 & 104 & \\
\hline Age range (mean) & 80 - 93 (83.8) & 75 - 79 (76.7) & \\
\hline \multicolumn{4}{|l|}{ Initial treatment } \\
\hline Radical standard & $83(80.6 \%)$ & $93(89.4 \%)$ & \multirow{7}{*}{ NS } \\
\hline RT alone & 27 & 16 & \\
\hline RT + chemotherapy & 26 & 26 & \\
\hline Operation & 23 & 35 & \\
\hline Operation + RT & 7 & 16 & \\
\hline Palliative & $8(7.8 \%)$ & $5(4.8 \%)$ & \\
\hline Untreated & $12(11.7 \%)$ & $6(5.8 \%)$ & \\
\hline \multicolumn{4}{|l|}{ Initial treatment (radical standard) } \\
\hline According our policy & 64 & 85 & $\mathrm{p}<0.01$ \\
\hline Compromised and modified treatment & 19 & 8 & \\
\hline \multicolumn{4}{|l|}{ Achievement of the planned initial treatment } \\
\hline Achievement & $77(84.6 \%)$ & $93(94.9 \%)$ & $\mathrm{p}<0.05$ \\
\hline Omission & 14 & 5 & \\
\hline \multicolumn{4}{|l|}{ Complication after treatment } \\
\hline Appearance (treatment-related death) & $24(4)$ & $19(0)$ & NS \\
\hline None & 67 & 78 & \\
\hline \multicolumn{4}{|l|}{ Prognosis } \\
\hline Disease specific 5 year survival rate & $35.8 \%$ & $53.9 \%$ & $\mathrm{p}<0.01$ \\
\hline Limited in case of the patients treated & & & \\
\hline according to our policy & $62.7 \%$ & $63.2 \%$ & NS \\
\hline
\end{tabular}

RT: radiotherapy; NS: not significance. 


\subsection{Complications after Treatment}

In the 91 cases that receive radical standard or palliative treatment, complications after treatment were identified in 24 cases (26.4\%). The most common was pneumonia (aspiration, postoperative, or interstitial), occurring in 13 cases (54.1\%). Others included sepsis, kidney failure, progression of dementia, cerebral infarction, fulminant hepatitis, disseminated intravascular coagulation, and chronic subdural hematoma, showing many serious complications. Four treatment-related deaths were recognized among these cases. On the other hand, in Group P, among the 98 cases that received radical standard or palliative treatment, complications after treatment were identified in 19 cases (19.4\%). As in the subject group, serious complications had occurred, but no treatmentrelated deaths were encountered. No significant difference in the rate of complications after treatment was evident between groups (Table 3).

\subsection{Prognosis}

Disease-specific 5-year survival rates were $35.8 \%$ in the subject group and 53.9\% in Group P (Figure 1), showing a significant difference between groups $(\mathrm{p}<0.01)$. However, when we limited analysis to radical standard treatment completed according to the policy of our institution, disease-specific 5-year survival rates were $62.7 \%$ in the subject group and 63.2\% in Group P, showing no significant difference between groups (Figure 2).

\section{Discussion}

Report of elderly individuals with head and neck cancer often consider patients around a cut-off of 70 or 75 years old and older [7]-[10]. Japanese people have among the longest life expectancies in the world, with an average lifespan of 84 years (men, 80 years; women, 87 years). Around the world, the number of countries with an average lifespan over 80 years old has expanded to 29 countries now. The average life expectancy for a 70-year-old Japanese is very long, at 19.5 years for women and 15.1 years for men, and that for an 80-year-old is 11.4 years for women and 8.5 years for men. On the basis of such information, when we examine elderly individuals with head and neck cancer, we think it appropriate to consider someone $\geq 80$ years old as elderly. In Japan, treatment and associated problems for head and neck cancer patients $\geq 80$ years old have been investigated in numerous studies [2] [3] [11]. The reason we chose a 75- to 79-year-old age group for comparison with the subject group was to clarify factors strongly influenced specifically by chronological age, as 75- to 79-yearold Japanese show no inferiority with younger patients for treatment choice, because Derks et al. [12] reported that they were able to provide standard treatment in 45- to 60-year-old head and neck cancer patients in 132 of 148 cases (89\%), the rate was equal to Group P (75- to 79-year-old) in 93 of 104 cases (89.4\% ).

Comparing head and neck cancer patients $\geq 80$ years old with 75 - to 79-year-olds showed no significant differences in background factors such as disease classification, comorbidities, multiple primary cancers, or PS between groups (Table 1). These factors were thus thought to be little influenced by chronological age. The ratio of males to females and the number of comorbidities are affected by chronological age. In Japan, the ratio of woman was $8.7 \%$ for 75 - 79 years old and $21.4 \%$ for $\geq 80$ years old. Because women have a longer average lifespan ( 87 years) than men ( 80 years old), the population differences between men and women $\geq 80$ years old are large (approximately 6.1 million women compared to approximately 3.2 million men [13]). Sarini et al. [10] pointed out that the proportion of women is increased among elderly patients with head and neck cancer, that the ratios of woman. The number of comorbidities is also increased among patients $\geq 80$ years old, with many cases showing more than three comorbidities, and conversely few cases showing none.

As for the disease-specific 5-year survival rate, a significant difference was seen between Group P (53.9\%) and the subject group (35.8\%). The proportion of patients receiving radical standard treatment did not differ significantly between groups. However, even if patients $\geq 80$ years old were classified as suitable to receive radical standard treatment, many cases were unable to complete treatment according to the policies of our institution. When disease-specific 5-year survival rates were determined for those patients able to be treated according to institutional policies, results were comparable (subject group, 62.7\%; Group P, 63.2\%). In addition, for patients who achieved the planned initial treatment, subject group was significantly low in comparison with $94.9 \%$ of p group in $84.6 \%$ in subject group. Because treatment according to our policy appears difficult to initiate for patients $\geq 80$ years old, and many of those patients who do start cannot complete treatment, the survival rates are poor. 
(\%) 100

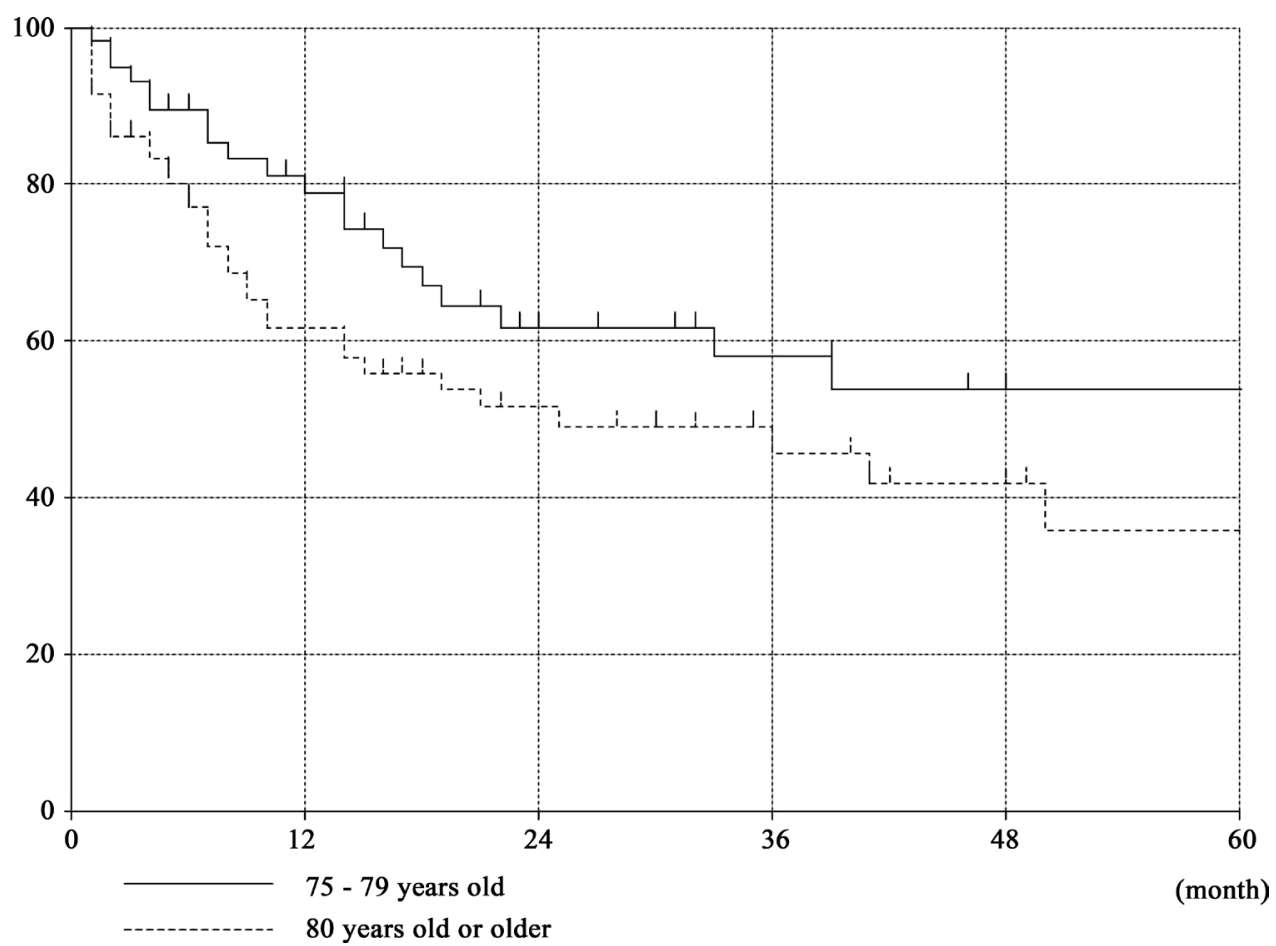

Figure 1. Disease specific survival rate in all cases.

(\%) 100

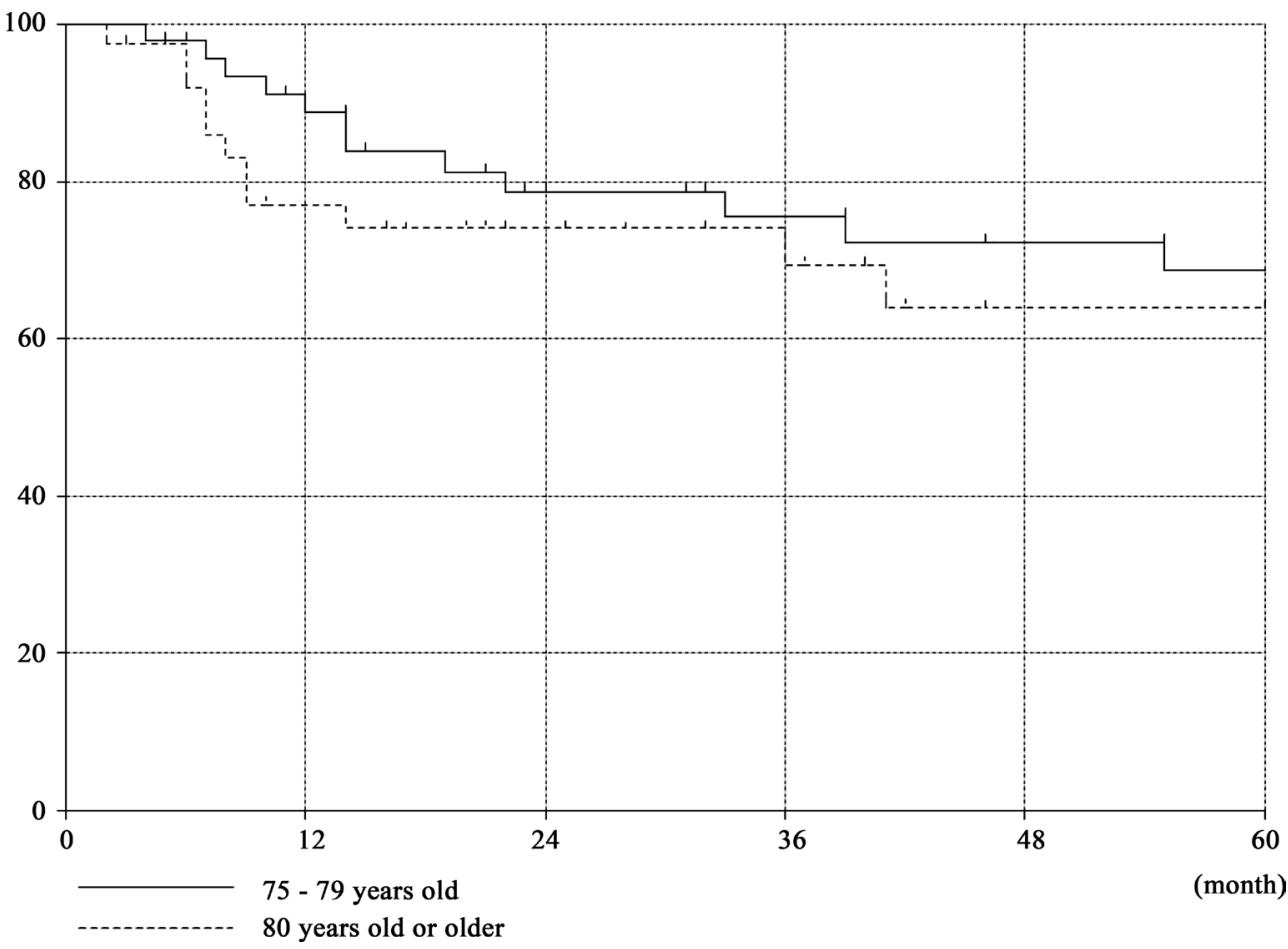

Figure 2. Disease specific survival rate in the limited cases treated according to the policy of our institution.

If an elderly patient $\geq 80$ years old can complete radical standard treatment, the prognosis seems comparable to that of a younger patient. Therefore, as noted in past reports [3] [11] [13], choice of treatment should not be based on chronological age, but instead simply according to standard policy where possible. Under the present conditions, we provided treatment just as wanted and could not choose it often. The reasons were oncological 
factors such as unresectable tumor or distant metastasis, refusal of treatment, and physical factors such as poor PS or number of comorbidities. Chaibi et al. [14] reported that cases in which the CIRS-G score is high typically cannot receive intensive treatment. Furthermore, Derks et al. [13] reported that they were able to provide standard treatment in 70- to 79-year-old head and neck cancer patients in 59 of 79 cases (75\%), compared to only 14 of 39 cases (36\%) among patients $\geq 80$ years old.

When a patient exceeds 80 years old, surpassing the average lifespan for a man, a feeling of satisfaction that the patient and family were able to accomplish some degree of longevity may arise. The desire for life may subsequently fade, and the patient may not accept the risk of complications that accompanies aggressive treatment to achieve radical cure and may instead decline treatment. When the patient and family do not expect to undergo aggressive treatment based on their outlook on life and death, sufficient information must be provided about symptoms and functional disorders that may arise in the future, and decisions on treatment policy need to be made. With regard to the drop in the acceptance of treatment among the elderly, Monden et al. [3] made similar points. Among patients $\geq 80$ years old who did not receive radical curative treatment, cases in which the patient or family declined aggressive treatment comprised $50 \%$. The will of the patient and family to undergo treatment may thus greatly influence decisions on treatment among elderly head and neck cancer patients $\geq 80$ years old. We need to make an effort to give elderly patients an incentive to undergo aggressive treatment.

It is necessary to pay full attention when we choose treatment for head and neck cancer patients $\geq 80$ years old, because complications after treatment can be serious and treatment-related death was recognized in four patients. Precise judgment of whether the patient can endure the treatment by examining physical function and comorbidities is crucial to limit the high incidence and severity of complications after treatment.

\section{Conclusions}

From January 2000 to December 2011, we evaluated 103 cases of head and neck cancer patients $\geq 80$ years old who visited our institution. We analyzed TNM stage, PS, multiple primary cancers, comorbidities, treatment, complications after treatment, and prognosis.

We think that the desire for treatment of the patient and family greatly influences decisions on treatment policy in head and neck cancer patients $\geq 80$ years old. We need to make an effort to give elderly patients an incentive to undergo aggressive treatment.

Treatment choices should be based on the wishes and motivations of the patient and the medical assessment of physical function. When a patient $\geq 80$ years old is treated, the high incidence of complications and severity of the disease should be considered.

\section{References}

[1] World Health Organization (WHO) in Internet, World Health Statistics 2014, Mortality and Burden of Disease, by Life Expectancy. http://apps.who.int/ghodata/

[2] Suzuki, M., Matayoshi, N., Hasegawa, M., et al. (2008) Treatment Outcomes in Head and Neck Cancer Patients Aged 80 or over. Japanese Journal of Head and Neck Cancer, 34, 594-599. http://dx.doi.org/10.5981/jjhnc.34.594

[3] Monden, S., Nishikawa, K., Morishita, T., et al. (2003) Head and Neck Cancer Treatment in the Elderly—Evaluation and Management of Complications. Nippon Jibiinkoka Gakkai Kaiho, 106, 7-16. http://dx.doi.org/10.3950/jibiinkoka.106.7

[4] Oken, M.M., Creech, R.H., Tormey, D.C., et al. (1982) Toxicity and Response Criteria of the Eastern Cooperative Oncology Group. American Journal of Clinical Oncology, 5, 649-655. http://dx.doi.org/10.1097/00000421-198212000-00014

[5] Charlson, M.E., Pompei, P., Ales, K.L., et al. (1987) A New Method of Classifying Prognostic Comorbidity in Longitudinal Studies: Development and Validation. Journal of Chronic Diseases, 40, 373-383. http://dx.doi.org/10.1016/0021-9681(87)90171-8

[6] Miller, M.D., Paradis, C.F., Houck, P.R., et al. (1992) Rating Chronic Medical Illness Burden in Geropsychiatric Practice and Research: Application of the Cumulative Illness Rating Scale. Psychiatry Research, 41, 237-248. http://dx.doi.org/10.1016/0165-1781(92)90005-N

[7] Milisavljević, D., Stanković, M., Zivić, M., et al. (2012) Head and Neck Cancer Surgery in Elderly: Complications and Survival Rate. Collegium Antropologicum, 36, 13-17.

[8] Van der Schroeff, M.P., Derks, W., Hordijk, G.J., et al. (2007) The Effect of Age on Survival and Quality of Life in 
Elderly Head and Neck Cancer Patients: A Long-Term Prospective Study. European Archives of Oto-Rhino-Laryngology, 264, 415-422. http://dx.doi.org/10.1007/s00405-006-0203-y

[9] Bernardi, D., Barzan, L., Franchin, G., et al. (2005) Treatment of Head and Neck Cancer in Elderly Patients: State of the Art and Guidelines. Critical Reviews in Oncology/Hematology, 53, 71-80. http://dx.doi.org/10.1016/j.critrevonc.2004.08.001

[10] Sarini, J., Fournier, C., Lefebvre, J.L., et al. (2001) Head and Neck Squamous Cell Carcinoma in Elderly Patients: A Long Term Retrospective Review of 273 Cases. Archives of Otolaryngology—Head \& Neck Surgery, 127, 1089-1092. http://dx.doi.org/10.1001/archotol.127.9.1089

[11] Saikawa, M., Ebihara, T. and Yoshidumi, T. (2000) Treatment for Head and Neck Cancer in Patients at the Age of 80 or Older. Journal of the Japan Broncho-Esophagological Society, 51, 93-97.

[12] Derks, W., Leeuw, R.D., Hordijk, G.J., et al. (2005) Reasons for Non-Standard Treatment in Elderly Patients with Advanced Head and Neck Cancer. European Archives of Oto-Rhino-Laryngology, 262, 21-26. http://dx.doi.org/10.1007/s00405-004-0744-x

[13] Statistics Japan in Internet, Ministry of Public Management, Estimate of the Population, by the Population for a Specific Age Group. http://www.stat.go.jp/index.htm

[14] Chaïbi, P., Magné, N., Breton, S., et al. (2011) Influence of Geriatric Consultation with Comprehensive Geriatric Assessment on Final Therapeutic Decision in Elderly Cancer Patients. Critical Reviews in Oncology/Hematology, 79, 302-307. http://dx.doi.org/10.1016/j.critrevonc.2010.08.004 